\title{
Incidental Lung Cancer of Explanted Lungs from Lung Transplant Recipients: Incidence, Characteristics, and 5-Year Survival
}

\author{
Yong Jun $\mathrm{Choi}^{1}$, Song Yee Kim ${ }^{1}$, Moo Suk Park ${ }^{1}$, Jin Gu Lee ${ }^{2}$, Hyo Chae Paik ${ }^{2}$, and Sang Hoon Lee \\ Departments of ${ }^{1}$ Internal Medicine, ${ }^{2}$ Thoracic and Cardiovascular Surgery, Yonsei University College of Medicine, Seoul, Korea.
}

Purpose: Recent history of malignancy without 5-year disease-free interval is an absolute contraindication for lung transplantation (LTx). However, in rare cases, lung cancer may be incidentally diagnosed in the explanted lung of recipients. We evaluated the prevalence, 5-year survival, and prognosis of incidental lung cancer after LTx.

Materials and Methods: Medical records of patients who underwent LTx at Severance Hospital between January 1, 2012 and June 30, 2019 were reviewed. Patients with incidental lung cancer were included, and those with histologically proven pre-transplant lung cancer were excluded.

Results: Of the 247 patients who underwent LTx, 6 (2.4\%) were diagnosed with incidental lung cancer. Interstitial lung disease (ILD) was the underlying lung disease in all patients. The median interval from the last preoperative computed tomography (CT) screening to LTx was 26 days. The most common histological type of incidental lung cancer was adenocarcinoma $(\mathrm{n}=4,66.7 \%)$. All Stage IV cases were misdiagnosed as fibrosis on preoperative chest CT. Patients with incidental lung cancer showed lower 5 -year survival than those without malignancy (median survival: 8.5 months vs. not reached, $p=0.047$, respectively). Patients with Stage III or IV demonstrated lower 5-year survival than those with Stage I or II and those without malignancy (median survival: 5 months, 19 months, and not reached, respectively, $p=0.011$ ).

Conclusion: Multidisciplinary preoperative screening and serial imaging studies within short intervals are required to differentiate lung malignancy from fibrotic foci. Furthermore, active pathologic examination of suspicious lung lesions is required in patients at high risk for lung cancer.

Key Words: Non-small cell lung cancer, lung transplantations, transplant recipient

\section{INTRODUCTION}

Since the first human lung transplantation (LTx) was performed in 1963, LTx has become a treatment option for various endstage lung diseases. ${ }^{1}$ According to a 2019 report from the Inter-

Received: July 2, 2020 Revised: August 13, 2020

Accepted: September 24, 2020

Corresponding author: Sang Hoon Lee, MD, PhD, Department of Internal Medicine, Severance Hospital, Yonsei University College of Medicine, 50-1 Yonsei-ro, Seodaemun-gu, Seoul 03722, Korea.

Tel: 82-2-2228-1930, Fax: 82-2-393-6884, E-mail: cloud9@yuhs.ac

-The authors have no potential conflicts of interest to disclose.

(C) Copyright: Yonsei University College of Medicine 2020

This is an Open Access article distributed under the terms of the Creative Commons Attribution Non-Commercial License (https://creativecommons.org/licenses/ by-nc/4.0) which permits unrestricted non-commercial use, distribution, and reproduction in any medium, provided the original work is properly cited. national Thoracic Organ Transplant Registry of the International Society for Heart and Lung Transplantation, the number of LTx has been continuously increasing and, consequently, the survival of adult recipients has been increasing; from 2010 to 2017, the median survival of these LTx recipients was 6.7 years. ${ }^{2}$ The most common indications for LTx include chronic obstructive pulmonary disease (COPD) and idiopathic pulmonary fibrosis (IPF), which account for $30.1 \%$ and $26.1 \%$ of LTx cases, respectively. ${ }^{2}$ These diseases are associated with a common risk factor for lung cancer-cigarette smoking; therefore, COPD and IPF are known as risk factors for lung cancer. Since COPD and lung cancer share underlying genetic predispositions, such as telomere shortening, mitochondrial dysfunction, and premature aging, the risk of squamous cell carcinoma is two times higher in COPD patients than in the general population. ${ }^{3}$ Similarly, IPF increases the risk of lung cancer from $7 \%$ to $20 \%$ through mul- 
tiple common genetic, molecular, and cellular processes. ${ }^{4}$

Except in cases of localized non-melanoma skin cancer, although a recent history of malignancy without a 5-year diseasefree interval is an absolute contraindication for LTx, the recipients with incidental lung cancer in the explanted lungs have already undergone LTx. ${ }^{5}$ In previous studies, the prevalence of incidental lung cancer has been reported to range from $0.8 \%$ to $2.9 \% .{ }^{6}$ The association between the stage and prognosis of incidental lung cancer is not well-established, although it is generally reported that the higher stages are associated with poor survival. ${ }^{6,7}$ Therefore, early detection of lung cancer is important in patients awaiting LTx for appropriate patient selection and improved LTx outcomes. To date, however, most relevant studies have been confined to non-Asian regions. Therefore, in this study, we aimed to evaluate the prevalence, characteristics, survival, and prognosis of incidental lung cancer after LTx in the Korean population and compare them with those reported in non-Asian populations.

\section{MATERIALS AND METHODS}

\section{Patients and study design}

We reviewed the medical records of all patients who underwent LTx at Severance Hospital between January 1, 2012, and June 30 , 2019. Patients with malignancy in the explanted lung were included, and those with histologically proven lung cancer before LTx were excluded. Moreover, patients who underwent retransplantation were also excluded (Fig. 1).

We divided the enrolled patients into two groups based on the presence of incidental lung cancer and compared the prevalence, characteristics, survival, and prognosis between them.

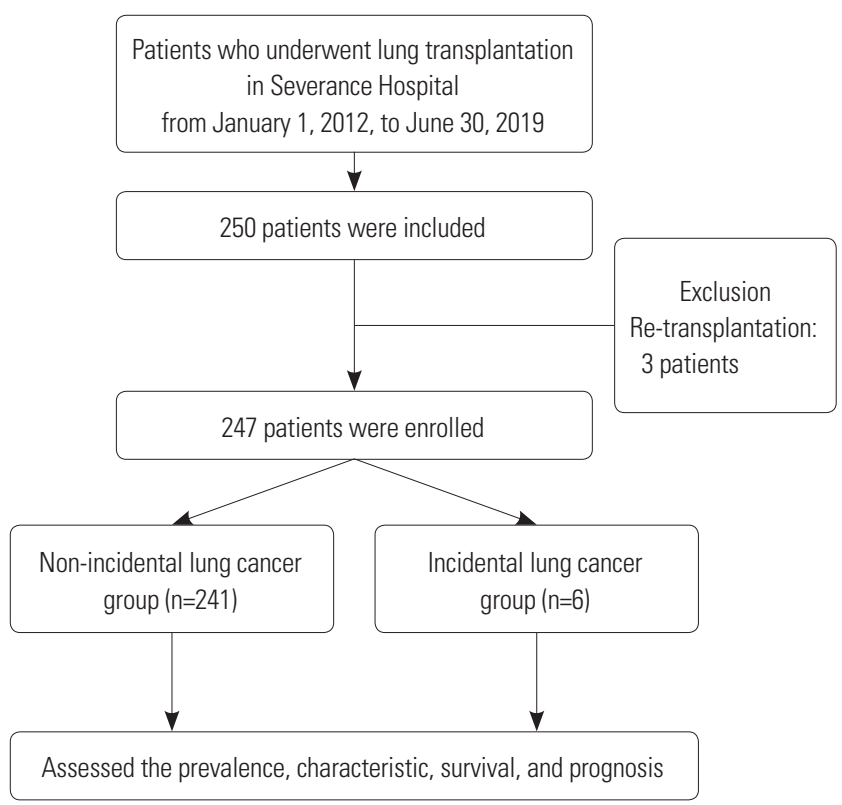

Fig. 1. Flowchart depicting the study design.

\section{Data collection}

We collected the data on baseline characteristics such as sex, age at LTx, smoking history, and the time of diagnosis and pathology of the underlying lung disease. Furthermore, information on the date and type of LTx, postoperative treatment, status of recurrence or progression, overall survival duration, death, and cause of death were collected.

All patients who were selected for LTx had undergone chest computed tomography (CT) as part of the preoperative screening. The date and findings of the two most recent CT scans before LTx were collected.

All explanted lungs underwent pathologic examination, and the pathologic stage of lung cancer was evaluated in accordance with the American Joint Committee on Cancer 8th edition TNM staging system.

\section{Outcome measures}

Recurrence was defined as radiological evidence [CT, positron emission tomography (PET), brain magnetic resonance imaging, or whole-body bone scan] or histological evidence of recurrent disease in patients with an operable stage (I, II, and IIIA). Progression was defined as the presence of radiological or histological evidence of worsening malignant disease in patients with an inoperable stage (IIIB/C and IV). The 5-year survival rates were calculated based on the duration between the transplantation and the last follow-up. Progression-free survival was defined as the duration between the date of transplantation and that of confirmation of recurrence or progression.

\section{Statistical analysis}

Statistical analysis was performed with R software (version 3.6.3; R Foundation for Statistical Computing, Vienna, Austria). To compare continuous variables, the Mann-Whitney U test was used for non-parametric variables, based on the normality assumptions from the Shapiro-Wilk test, and the results are presented as the median and interquartile range. The chi-square test or Fisher's exact test was used to compare categorical variables, and the results are presented as number and frequency. Kaplan-Meier curves were plotted to evaluate the cumulative 5 -year survival rates. $p$-values $<0.05$ were considered statistically significant.

\section{Ethics}

This study was approved (approval no. 4-2020-2) by the Institutional Review Board of Severance Hospital. The requirement for informed consent was waived due to the retrospective nature of this study. 


\section{RESULTS}

\section{Baseline characteristics and incidence of explanted lung cancers}

Among 247 patients who underwent LTx, 6 (2.4\%) were diagnosed with explanted lung cancer at the time of transplantation. The baseline characteristics of these patients are summarized in Table 1. The most common causes of LTx included interstitial lung disease (ILD) ( $\mathrm{n}=177 ; 71.7 \%)$, post-stem cell transplantation bronchiolitis obliterans $(\mathrm{n}=18 ; 7.3 \%)$, bronchiectasis $(\mathrm{n}=$ 14; $5.7 \%)$, and COPD ( $\mathrm{n}=11 ; 4.4 \%)$. Almost all of the patients underwent bilateral LTx ( $\mathrm{n}=236 ; 95.6 \%)$, whereas only 11 patients underwent single-LTx (right-LTx, $\mathrm{n}=10,4.0 \%$; left-LTx, $\mathrm{n}=1,0.4 \%)$.

\section{Radiologic findings}

All patients with lung cancer underwent chest CT, and the interval between the last pre-transplant $\mathrm{CT}$ and transplantation ranged from 8 to 44 days (median 26 days). The findings from imaging investigations are described in Fig. 2 and summarized in Table 2. Five patients were diagnosed with IPF, four had the usual interstitial pneumonia (UIP) pattern, and one had a probable IPF pattern on chest CT. In cases of probable IPF, the IPF was diagnosed by using a multidisciplinary approach that comprised the subdisciplines of pulmonology, cardiothoracic surgery, radiology, and pathology. ${ }^{8}$ On the pre-transplantation review for incidental cancer lesions on chest $\mathrm{CT}$ in the six patients,
Patients B, D, and F had patterns of ILD exacerbation with pneumonia, whereas patients A and E primarily had a pneumonia and an exacerbation pattern, respectively. Only Patient $\mathrm{C}$ had a growing nodular lesion.

\section{Pathology and TNM stage of explanted lung cancer} All patients with explanted lung cancer had ILD on the pathological analysis of the explanted lung: five had a UIP pattern and one had a non-specific interstitial pneumonia pattern. With regard to the pathology of lung cancer lesions, the most common cancer was adenocarcinoma $(n=4 ; 66.6 \%)$, followed by squamous cell carcinoma ( $\mathrm{n}=1 ; 16.7 \%)$, and carcinoid tumors $(\mathrm{n}=1$; 16.7\%) (Table 3). On TNM staging, three cases were identified as $\mathrm{T} 4$, and the largest and smallest incidental cancers were 10 $\mathrm{cm}$ and $0.3 \mathrm{~cm}$ in maximum diameter, respectively. Stage IA was identified in two cases, whereas stages IIB, IIIB, and IV were identified in one, one, and two patients, respectively. In patients with Stage IV (E, F) cancer, preoperative chest CT showed fibrotic foci with a pattern of ILD exacerbation rather than a malignant mass, and it was also accompanied by reactive lymph node enlargement in Patient $\mathrm{F}$.

\section{Prognosis and survival}

The overall 5-year survival rate was $58.7 \%$. The cumulative 5 -year survival rates of the incidental lung cancer group and non-incidental lung cancer group are shown in Supplementary Fig. 1A (only online). There was a significant difference between the

Table 1. Baseline Characteristics of All Patients

\begin{tabular}{|c|c|c|c|c|}
\hline Variables & Total $(n=247)$ & Non-incidental lung cancer ( $\mathrm{n}=241)$ & Incidental lung cancer ( $n=6)$ & $p$ value \\
\hline Age (median age) & $56.0(46.0-62.0)$ & $56.0(46.0-62.0)$ & $58.5(53.0-62.0)$ & 0.538 \\
\hline Sex & & & & 1.000 \\
\hline Male & $156(63.2)$ & $152(63.1)$ & $4(66.7)$ & \\
\hline Female & $91(36.8)$ & 89 (36.9) & $2(33.3)$ & \\
\hline Transplantation type & & & & 0.866 \\
\hline Bilateral lung & $236(95.6)$ & $230(95.5)$ & $6(100.0)$ & \\
\hline Right lung & $10(4.0)$ & $10(4.1)$ & $0(0.0)$ & \\
\hline Left lung & $1(0.4)$ & $1(0.4)$ & $0(0.0)$ & \\
\hline Cause of transplantation & & & & 0.965 \\
\hline $\mathrm{BE}$ & $14(5.7)$ & $14(5.8)$ & $0(0.0)$ & \\
\hline COPD & $11(4.4)$ & $11(4.6)$ & $0(0.0)$ & \\
\hline ILD & $177(71.7)$ & $171(71.0)$ & $6(100.0)$ & \\
\hline IPAH & $5(2.0)$ & $5(2.0)$ & $0(0.0)$ & \\
\hline LAM & $5(2.0)$ & $5(2.0)$ & $0(0.0)$ & \\
\hline Post-SCT BO & $18(7.3)$ & $18(7.5)$ & $0(0.0)$ & \\
\hline TDL & $4(1.6)$ & $4(1.7)$ & $0(0.0)$ & \\
\hline Others & $13(5.3)$ & $13(5.4)$ & $0(0.0)$ & \\
\hline \multicolumn{5}{|l|}{ Stage } \\
\hline | or II & & NA & $3(50.0)$ & \\
\hline III or IV & & NA & $3(50.0)$ & \\
\hline
\end{tabular}

BE, bronchiectasis; COPD, chronic obstructive pulmonary disease; ILD, interstitial lung disease; IPAH, idiopathic pulmonary arterial hypertension; LAM, Iymphangioleiomyomatosis; post-SCT BO, post-stem cell transplantation bronchiolitis obliterans; TDL, tuberculosis-destroyed lung. 
two groups in the cumulative survival (5-year survival rate: $16.7 \%$ vs. $59.8 \%$; median survival: 8.5 vs. not reached, respectively, $p=$ 0.047). When the incidental lung cancer group was divided into two groups based on the cancer stages, the group with Stage
III or IV cancer demonstrated significantly lower 5-year survival than those with Stage I or II cancer and those without incidental lung cancer (5-year survival rate: 59.8\%, 33.3\%, and $16.7 \%$; median survival: 5,19 , and not reached, respectively, $p=0.011$ )
Chest CT 1

A

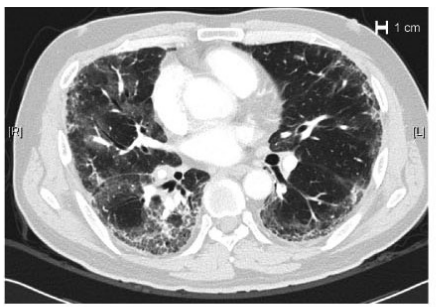

B

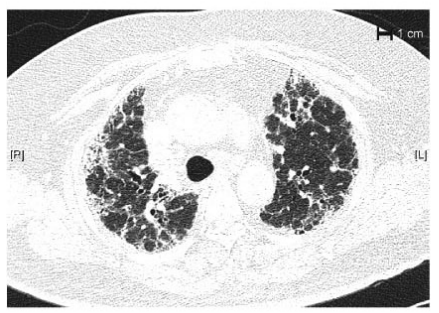

C

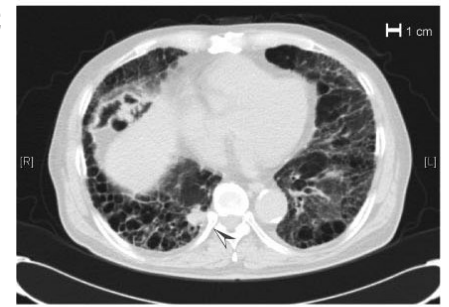

D

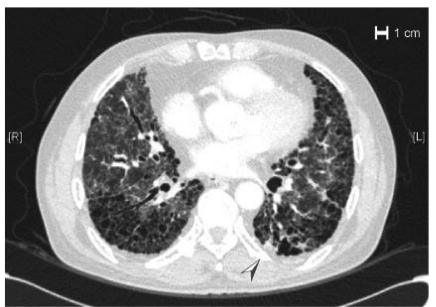

E

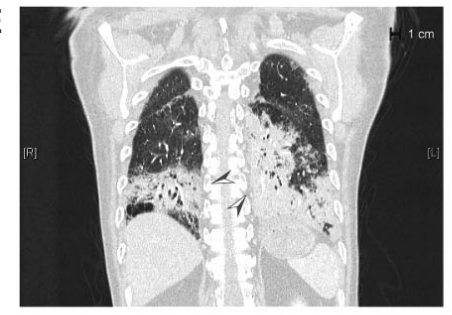

$\mathbf{F}$

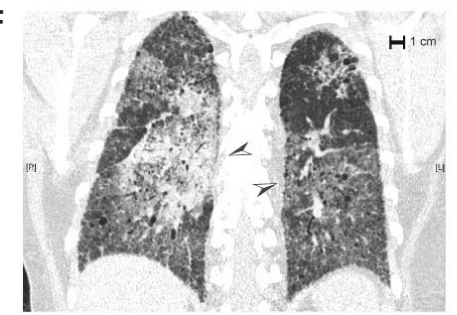

Chest CT 2
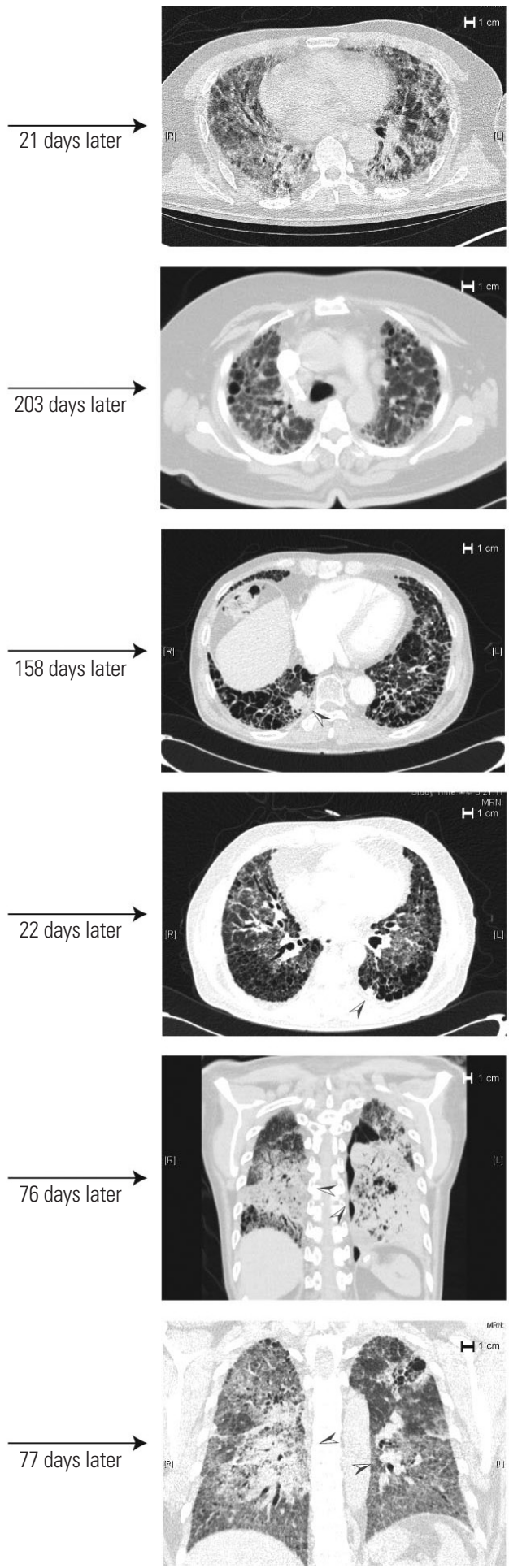

Post LTx chest CT
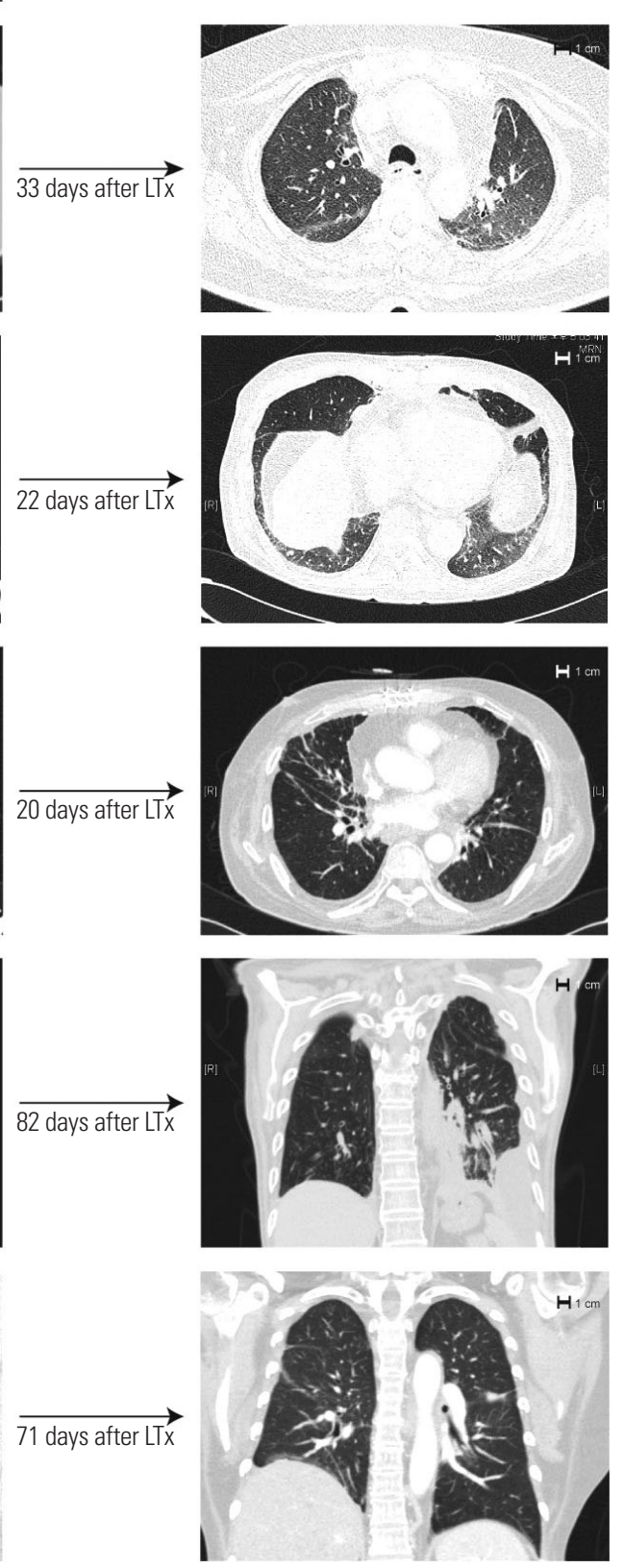

Fig. 2. Chest CT images of the patients with incidental lung cancer. CT 1 and CT 2 were defined as the two most recent CTs performed prior to surgery. The arrow marks malignant lesions. $(A)$ and $(B)$ demonstrated no definite malignancy, $(C)$ and $(D)$ demonstrated suspicious focal malignancy, $(\mathrm{E})$ and (F) demonstrated diffuse malignancy in fibrotic lesion. CT, computed tomography; LTx, lung transplantation. 
Table 2. Radiologic Findings of Incidental Lung Cancer in Lung Transplant Recipients

\begin{tabular}{|c|c|c|c|}
\hline \multirow{2}{*}{ Patients } & Chest CT $1^{*}$ & Chest CT $2^{*}$ & \multirow{2}{*}{ Interval to LTx (days) } \\
\hline & Finding & Finding & \\
\hline A & Probable IPF & Probable IPF with pneumonitis & 42 \\
\hline B & UIP & Progressive UIP with acute exacerbation and combined pneumonia & 17 \\
\hline C & UIP with right lower lobe subpleural nodule & UIP with growing right lower lobe subpleural nodule & 44 \\
\hline D & UIP & Exacerbation of UIP, with focal pneumonia & 10 \\
\hline$E$ & UIP & Exacerbation of UIP & 8 \\
\hline $\mathrm{F}$ & NSIP & $\begin{array}{l}\text { Progressive NSIP with bronchopneumonia, enlarged lymph nodes in } \\
\text { the bilateral hilar and paratracheal area }\end{array}$ & 35 \\
\hline
\end{tabular}

CT, computed tomography; LTx, lung transplantation; IPF, idiopathic pulmonary fibrosis; UIP, usual interstitial pneumonia; NSIP, non-specific interstitial pneumonia.

${ }^{*}$ CT 1 and CT 2 were defined as the two most recent CTs performed prior to surgery.

Table 3. Pathologic Findings of Incidental Lung Cancer in Lung Transplant Recipients

\begin{tabular}{|c|c|c|c|c|c|}
\hline \multirow{2}{*}{ Patients } & \multirow{2}{*}{$\begin{array}{c}\text { Whole lung } \\
\text { Pathology }\end{array}$} & \multicolumn{4}{|c|}{ Lung cancer lesion } \\
\hline & & Pathology & Maximum diameter (cm) & Location & Stage (TNM) \\
\hline A & UIP & Adenocarcinoma, acinar type & Multiple & RUL, RML, RLL & IIIB (pT4N3M0) \\
\hline B & UIP & Typical carcinoid tumor & 0.3 & LUL & IA (T1aNOMO) \\
\hline C & UIP & Invasive adenocarcinoma, acinar predominant & 2.5 & RLL & IIB (pT2aN1M0) \\
\hline D & UIP & Squamous cell carcinomas & 0.5 & LLL & IA (pT1aNOMO) \\
\hline $\mathrm{E}$ & UIP & Invasive mucinous adenocarcinoma & Right: 10 / left: 10 & RUL, RML, RLL, LUL, LLL & IVA (pT4N3M1a) \\
\hline $\mathrm{F}$ & CFIP & Adenocarcinoma, invasive acinar predominant & Right: 10 / left: 10 & RUL, RML, RLL, LUL, LLL & IVB (pT4N1aM1c) \\
\hline
\end{tabular}

UIP, usual interstitial pneumonia; CFIP, chronic fibrosing interstitial pneumonia; RUL, right upper lobe; RML, right middle lobe; RLL, right lower lobe; LUL, left upper lobe; LLL, left lower lobe.

Table 4. Post-Transplant Treatment and Prognoses of Cancer Patients

\begin{tabular}{|c|c|c|c|c|c|c|}
\hline Patient & Metastasis at diagnosis & Cancer therapy & $\begin{array}{c}\text { Duration of cancer } \\
\text { therapy (months) }\end{array}$ & Cause of death & Immunosuppressive agent & Progression site \\
\hline$A$ & None & None & & Pneumonia & MMF, tacrolimus & None \\
\hline B & None & None & & Pneumonia & MMF, tacrolimus & None \\
\hline C & None & None & & Drug induced TTP & MMF, tacrolimus & None \\
\hline D & None & None & & Alive & MMF, tacrolimus & None \\
\hline$E$ & Pleura & None & & Cancer progression & MMF, tacrolimus & Bone \\
\hline $\mathrm{F}$ & Bone, brain & Afatinib & 6 & Cancer progression & MMF, tacrolimus & Brain \\
\hline
\end{tabular}

TTP, thrombotic thrombocytopenic purpura; MMF, mycophenolate mofetil.

(Supplementary Fig. 1B, only online).

No recurrence was observed in this study. Progression was observed in two cases and occurred in all Stage IV patients (Table 4$)$. The most common causes of death were pneumonia $(\mathrm{n}=$ $2 ; 33.3 \%)$ and cancer progression $(\mathrm{n}=2 ; 33.3 \%)$.

\section{DISCUSSION}

In this study, the prevalence of incidental lung cancer in the explanted lung was $2.4 \%$, which was similar to that reported in previous studies. ${ }^{6}$ However, all patients with explanted lung cancer had underlying IPF in the present study, which was a different finding compared to previous studies. Panchabhai, et al. ${ }^{9}$ reported that, among a total of 24 patients with incidental lung cancer, 16 (66.7\%) had ILD and 8 (33.3\%) had COPD. Similar- ly, Strollo, et al. ${ }^{10}$ reported that 11 (50\%) and 10 (45.5\%) patients in their study sample of 22 patients with incidental lung cancer had COPD and ILD, respectively. The absence of COPD patients in the present study is attributed to the LTx trend in Korea. Unlike in other countries, a considerably greater number of LTx are performed for IPF than for COPD in Korea. According to the Korean Network for Organ Sharing (KONOS), in 2019, among a total of 92 LTx, IPF accounted for 41 (44.6\%) cases; in 2018, emphysema accounted for only 1 (1.9\%) LTx case. Another possibility is that lung nodules are more easily distinguishable on preoperative screening in COPD patients than in ILD patients, which may have resulted in fewer incidental lung cancers being found intraoperatively during LTx in COPD patients. ${ }^{11}$

IPF is a well-known risk factor of lung cancer. Several studies have reported the prevalence of lung cancer in IPF patients in the range of 3.0-45.7\%, with an approximately five times high- 
er risk than the general population. ${ }^{12-14}$ In an autopsy study, the prevalence of lung cancer in IPF patients was $48.2 \%$, suggesting that many cases of lung cancer in IPF patients remain undiagnosed..$^{15}$ One of the causes for this is that the underlying IPF occasionally makes it difficult to differentiate between lung malignancy and fibrotic foci, ${ }^{9}$ and this misdiagnosis is more frequent in patients with end-stage lung disease or a flare-up of IPF that requires LTx.

Despite various preoperative screening imaging modalities, including thoracic high-resolution CT, incidental cancers in the explanted lung are observed in $1-3 \%$ of all LTx cases. ${ }^{16}$ In most of the incidental lung cancer patients in this study, it was difficult to distinguish between cancer and exacerbated lesions, even in Stage IV cancer patients. These difficulties have been reported in a previous study wherein radiologists, who were blinded to the pathologic diagnosis of recipients, reviewed preoperative screening images of patients with incidental lung cancer. ${ }^{10}$ However, they could not confidently identify malignancy in 12 (54.5\%) of a total of 22 patients. Recently, LTx has been suggested as an effective treatment for patients with early lung cancer. Glanville and Wilson ${ }^{17}$ recommended screening imaging studies to be conducted every 3 months to ascertain changes in the cancer stage in patients who are awaiting transplantation for non-small cell lung cancer. Similarly, we can also make similar recommendation that, for the early detection of lung cancer, chest imaging should be performed in patients who are at high risk for lung cancer at least once every 3 months while awaiting LTx. However, this recommendation needs to be validated through further research. PET alone may not be helpful for detecting malignancies due to many false-positive results secondary to inflammatory changes, although a combination of PET with chest CT may facilitate the evaluation of malignancies, especially in cases with distant metastasis. Furthermore, the identification of tumor markers may be helpful in the preoperative diagnosis of incidental lung cancers in patients at high risk for lung cancer.

In addition to preoperative imaging, preoperative pathological examination of the suspected lesions is also important. However, biopsies of suspected lesions can be dangerous, as invasive procedures may result in pneumothorax, empyema, pneumonia, and respiratory insufficiency, which are critically injurious to patients with end-stage IPF ${ }^{18}$ In comparison, endobronchial ultrasound-guided bronchoscopic biopsy of suspicious lung lesions or lymph nodes has been considered to be a less invasive diagnostic modality in patients with end-stage IPF. ${ }^{19}$

In a recent literature review of 13 articles on explanted lung cancer, the most common type of histologic cancer was adenocarcinoma ( $\mathrm{n}=51,48.6 \%)$, followed by squamous cell carcinoma $(\mathrm{n}=24,22.9 \%) .^{9}$ Similarly, in the present study, adenocarcinoma and squamous carcinoma were identified in four patients (66.6\%) and one patient (16.7\%), respectively.

According to a 2019 KONOS report, the cumulative 5-year survival rate of all LTx recipients in Korea was $48.3 \%$. In the present study, the cumulative 5-year survival rates in all patients, non-incidental lung cancer patients, and incidental cancer patients were $58.7 \%, 59.8 \%$, and $16.7 \%$, respectively. Furthermore, the stage of incidental lung cancer correlated with the overall survival. Most previous studies have reported that a lower stage is associated with a higher survival rate. According to a previous study by de Perrot, et al., ${ }^{7}$ the 5-year survival rate was significantly higher in patients with Stage I cancer (51\%) than in those with Stages II and III cancer (14\%). Similarly, we observed a negative association between the stage of lung cancer and 5-year survival rate in the present study.

Furthermore, the prognosis of incidental lung cancer in LTx recipients is related to the appropriate treatment. ${ }^{20}$ However, the therapeutic strategy in incidental lung cancer has not been well-established. Only some case series have described adjuvant chemotherapy in incidental lung cancer at the $\mathrm{T} 2 \mathrm{~N} 1$ stage; however, the effect of treatment was still unclear. ${ }^{20}$ Moreover, targeted therapies can be used in the presence of specific mutations.

Despite inadequate clinical data, the post-LTx use of immunosuppressive agents may cause the progression or recurrence of incidental lung cancer. ${ }^{9}$ Especially, calcineurin inhibitors have been reported to be associated with tumor progression through various mechanisms, such as the disruption of apoptosis and DNA repair. ${ }^{21,22}$ However, mTOR inhibitors have been observed to decrease cancer progression in recipients of kidney transplantation. ${ }^{23,24}$ Therefore, an individualized post-LTx immunosuppression strategy is necessary, and further studies are required to develop the criteria to guide individualized therapy.

The present study had some limitations. Mainly, since the size of the study sample was small, the results cannot be generalized. A multicenter study with a larger sample is required to validate the results of this study. In addition, the retrospective design of this study may have resulted in some unexpected bias in the investigative process.

In conclusion, similar to the results of previous non-Asian studies, the prevalence of incidental lung cancer in LTx recipients in this study was $2.4 \%$. The 5 -year survival rate of LTx recipients with incidental lung cancer was significantly lower than that of people without cancer. To reduce the incidence of incidental lung cancer, a multidisciplinary review of the preoperative screening studies, as well as serial imaging studies, are required. Furthermore, active histopathological examination of suspicious lung lesions is required to identify malignancy.

\section{AUTHOR CONTRIBUTIONS}

Conceptualization: Sang Hoon Lee and Yong Jun Choi. Data curation: Yong Jun Choi. Formal analysis: Yong Jun Choi and Sang Hoon Lee. Investigation: all authors. Methodology: Yong Jun Choi and Sang Hoon Lee. Project administration: Song Yee Kim. Resources: Song Yee Kim, Moo Suk Park, Jin Gu Lee, and Hyo Chae Paik. Software: Yong Jun Choi and Sang Hoon Lee. Supervision: Song Yee Kim, Moo Suk Park, Jin Gu Lee, Hyo Chae Paik, and Sang Hoon Lee. Validation: Yong Jun Choi. Visualization: Yong Jun Choi. Writing_original draft: 
Yong Jun Choi and Sang Hoon Lee. Writing_review \& editing: Yong Jun Choi and Sang Hoon Lee. Approval of final manuscript: all authors.

\section{ORCID iDs}

$\begin{array}{ll}\text { Yong Jun Choi } & \text { https://orcid.org/0000-0002-6114-2059 } \\ \text { Song Yee Kim } & \text { https://orcid.org/0000-0001-8627-486X } \\ \text { Moo Suk Park } & \text { https://orcid.org/0000-0003-0820-7615 } \\ \text { Jin Gu Lee } & \text { https://orcid.org/0000-0003-2767-6505 } \\ \text { Hyo Chae Paik } & \text { https://orcid.org/0000-0001-9309-8235 } \\ \text { Sang Hoon Lee } & \text { https://orcid.org/0000-0002-7706-5318 }\end{array}$

\section{REFERENCES}

1. Hardy JD, Webb WR, Dalton ML Jr, Walker GR Jr. Lung homotransplantation in man. JAMA 1963;186:1065-74.

2. Chambers DC, Cherikh WS, Harhay MO, Hayes D Jr, Hsich E, Khush KK, et al. The International Thoracic Organ Transplant Registry of the International Society for heart and lung transplantation: thirty-sixth adult lung and heart-lung transplantation Report-2019; focus theme: donor and recipient size match. J Heart Lung Transplant 2019;38:1042-55.

3. Young RP, Duan F, Chiles C, Hopkins RJ, Gamble GD, Greco EM, et al. Airflow limitation and histology shift in the national lung screening trial. The NLST-ACRIN Cohort Substudy. Am J Respir Crit Care Med 2015;192:1060-7.

4. Park J, Kim DS, Shim TS, Lim CM, Koh Y, Lee SD, et al. Lung cancer in patients with idiopathic pulmonary fibrosis. Eur Respir J 2001;17:1216-9.

5. Weill D. Lung transplantation: indications and contraindications. J Thorac Dis 2018;10:4574-87.

6. Ahmad U, Hakim AH, Tang A, Tong MZ, Bribriesco A, Budev M, et al. Patterns of recurrence and overall survival in incidental lung cancer in explanted lungs. Ann Thorac Surg 2019;107:891-6.

7. de Perrot M, Chernenko S, Waddell TK, Shargall Y, Pierre AF, Hutcheon $\mathrm{M}$, et al. Role of lung transplantation in the treatment of bronchogenic carcinomas for patients with end-stage pulmonary disease. J Clin Oncol 2004;22:4351-6.

8. Raghu G, Remy-Jardin M, Myers JL, Richeldi L, Ryerson CJ, Lederer DJ, et al. Diagnosis of idiopathic pulmonary fibrosis. An official ATS/ERS/JRS/ALAT clinical practice guideline. Am J Respir Crit Care Med 2018;198:e44-68.

9. Panchabhai TS, Arrossi AV, Patil PD, Bandyopadhyay D, Budev MM, McCurry KR, et al. Unexpected neoplasms in lungs explanted from lung transplant recipients: a single-center experience and review of literature. Transplant Proc 2018;50:234-40.

10. Strollo DC, Dacic S, Ocak I, Pilewski J, Bermudez C, Crespo MM.
Malignancies incidentally detected at lung transplantation: radiologic and pathologic features. AJR Am J Roentgenol 2013;201:10816.

11. Grewal AS, Padera RF, Boukedes S, Divo M, Rosas IO, Camp PC, et al. Prevalence and outcome of lung cancer in lung transplant recipients. Respir Med 2015;109:427-33.

12. Ozawa Y, Suda T, Naito T, Enomoto N, Hashimoto D, Fujisawa T, et al. Cumulative incidence of and predictive factors for lung cancer in IPF. Respirology 2009;14:723-8.

13. Kato E, Takayanagi N, Takaku Y, Kagiyama N, Kanauchi T, Ishiguro $\mathrm{T}$, et al. Incidence and predictive factors of lung cancer in patients with idiopathic pulmonary fibrosis. ERJ Open Res 2018;4: 00111-2016.

14. Hubbard R, Venn A, Lewis S, Britton J. Lung cancer and cryptogenic fibrosing alveolitis. A population-based cohort study. Am J Respir Crit Care Med 2000;161:5-8.

15. Matsushita H, Tanaka S, Saiki Y, Hara M, Nakata K, Tanimura S, et al. Lung cancer associated with usual interstitial pneumonia. Pathol Int 1995;45:925-32.

16. Mathew J, Kratzke RA. Lung cancer and lung transplantation: a review. J Thorac Oncol 2009;4:753-60.

17. Glanville AR, Wilson BE. Lung transplantation for non-small cell lung cancer and multifocal bronchioalveolar cell carcinoma. Lancet Oncol 2018;19:e351-8.

18. Van Raemdonck D, Vos R, Yserbyt J, Decaluwe H, De Leyn P, Verleden GM. Lung cancer: a rare indication for, but frequent complication after lung transplantation. J Thorac Dis 2016;8:S915-24.

19. Van Raemdonck DE. Editorial commentary: a new set of lungs or a new pair of glasses? Semin Thorac Cardiovasc Surg 2015;27:15-6.

20. Olland AB, Falcoz PE, Santelmo N, Kessler R, Massard G. Primary lung cancer in lung transplant recipients. Ann Thorac Surg 2014; 98:362-71.

21. Ahlers C, Kreideweiss S, Nordheim A, Rühlmann A. Cyclosporin A inhibits Ca2+-mediated upregulation of the DNA repair enzyme DNA polymerase beta in human peripheral blood mononuclear cells. Eur J Biochem 1999;264:952-9.

22. Pritchard DE, Singh J, Carlisle DL, Patierno SR. Cyclosporin A inhibits chromium(VI)-induced apoptosis and mitochondrial cytochrome c release and restores clonogenic survival in $\mathrm{CHO}$ cells. Carcinogenesis 2000;21:2027-33.

23. Chiurchiu C, Carreño CA, Schiavelli R, Petrone H, Balaguer C, Trimarchi $\mathrm{H}$, et al. Results of the conversion to everolimus in renal transplant recipients with posttransplantation malignancies. Transplant Proc 2010;42:277-9.

24. Fernández A, Marcén R, Pascual J, Galeano C, Ocaña J, Arellano EM, et al. Conversion from calcineurin inhibitors to everolimus in kidney transplant recipients with malignant neoplasia. Transplant Proc 2006;38:2453-5. 